\title{
EXPERIÊNCIAS EXEMPLARES DE DIVULGAÇÃO CIENTÍFICA DO CENTRO BRASILEIRO DE PESQUISAS FÍSICAS
}

MÁRCIA DE OLIVEIRA REIS BRANDÃO Universidade Federal Fluminense (UFF), Niterói, Rio de Janeiro, Brasil

E-mail:mreis@cbpf.br

LARISSA SANTIAGO ORMAY Universidade Federal do Rio de Janeiro (UFRJ) Rio de Janeiro, Brasil E-mail: larissa@cbpf.br 


\title{
EXPERIÊNCIAS EXEMPLARES DE DIVULGAÇÃO CIENTÍFICA DO CENTRO BRASILEIRO DE PESQUISAS FÍSICAS
}

Resumo: Este trabalho apresenta uma análise de três projetos bem sucedidos de divulgação científica desenvolvidos pelo Centro Brasileiro de Pesquisas Físicas (CBPF), unidade de pesquisa do Ministério da Ciência, Tecnologia e Inovação (MCTI) que é um dos institutos mais tradicionais do país. São eles: Desafios da Física, Algumas razões para ser um cientista e Laboratório Didático.

Palavras-chave: Divulgação científica; Educação; Ensino e Pesquisa em C\&T; Física; CBPF.

\section{EJEMPLOS DE EXPERIENCIAS EN COMUNICACIÓN DE LA CIENCIA DEL CENTRO BRASILEÑO DE INVESTIGACIONES FÍSICAS}

Resumen: Este artículo presenta un análisis de tres proyectos de comunicación científica exitosas desarrolladas por el Centro Brasileño de Investigaciones Físicas (CBPF), la unidad de investigación del Ministerio de Ciencia, Tecnología e Innovación (MCTI), que es uno de los institutos más tradicionales del país. Los proyectos son los siguientes: Desafíos físicos, Razones para ser un científico y Laboratorio Didáctico.

Palabras clave: Comunicación de la ciencia; Educación; Enseñanza e Investigación en S\&T Física; CBPF.

\section{EXAMPLES OF EXPERIMENTS FOR SCIENTIFIC DISSEMINATION OF THE BRAZILIAN CENTER FOR PHYSICS RESEARCH}

\begin{abstract}
This paper presents an analysis of three successful science communication projects developed by the Brazilian Center for Physics Research (CBPF), a research unit of the Ministry of Science, Technology and Innovation (MCTI) which is one of the most traditional institutes of the country. The projects are: Physical Challenges, Reasons to be a scientist and Didactic Laboratory.
\end{abstract}

Key-words: Popularization of Science, Education, Teaching and Research in S\&T; Physics; CBPF. 


\section{DivulgaÇÃo CIENTÍFICA, UM CONCEITO A SER EXPLORADO}

O presente trabalho objetiva analisar três projetos de divulgação científica do Centro Brasileiro de Pesquisas Físicas (CBPF), unidade de pesquisa do Ministério da Ciência, Tecnologia e Inovação do Brasil (MCTI). Porém, para melhor situar esse escopo nas discussões a respeito do tema, convém tecer algumas considerações preambulares sobre o próprio conceito de divulgação científica.

$O$ ato de divulgação, em si, pode significar uma série de espécies de comunicação de conteúdos. Em ciência, a própria publicação de artigos científicos em revistas e periódicos, a rigor, é um tipo de divulgação do fazer científico. No entanto, a expressão largamente utilizada no Brasil "divulgação científica" encerra um conceito particular: trata-se da difusão do conhecimento científico para públicos não especializados (MASSARANI, 2002). O termo "popularização da ciência" possui o mesmo sentido, sendo mais empregado na tradição de países anglo-saxônicos, a partir da década de 1950. "Vulgarização da ciência" (vulgarisation de la science) é a denominação mais usada na França e há, ainda, o termo "educação em ciência" (science education), que áreas como a Educação e a Museologia empregam com mais recorrência.

Contudo, de acordo com Silva e Carneiro (2008), ainda não existe consenso quanto ao significado dos termos difusão, disseminação, divulgação e popularização no Brasil, pois, frequentemente, todos esses vocábulos são usados com a mesma significação.

É de observar também que o que se chama de divulgação científica compreende um conjunto tão variado de textos e atividades, que todas as tentativas de definição e categorização a-históricas acabam malogradas. Por isso, o termo divulgação científica, longe de designar um tipo muito específico de conteúdo, está relacionado mais à forma como o conhecimento científico é produzido do que como é formulado, e como circula em uma determinada sociedade (SILVA, 2006: 53).

Com cada vez mais reflexões acadêmicas a respeito, seja na área da Comunicação Social ou da Ciência da Informação, a divulgação científica vem se desenvolvendo como uma nova disciplina, ainda em construção epistêmica. Por outro lado, a popularização da ciência e da tecnologia (C\&T) pode ser considerada, no âmbito de diversos países, como uma área aplicada, que está promovendo o desenvolvimento de programas e projetos de caráter 
EXPERIÊNCIAS EXEMPLARES DE DIVULGAÇÃO CIENTÍFICA DO CENTRO BRASILEIRO DE PESQUISAS FÍSICAS

nacional e transnacional e que está determinando ações específicas dos governos. Nesse contexto, como destacado por Pedro Leitão e Sarita Albagli (1997), tal mobilização das sociedades e dos seus governos se faz possível por dois elementos centrais: o histórico de experiências de divulgação científica com o qual esses países contam e a relevância que a popularização da C\&T adquire na agenda e no discurso político.

\section{Elemento de inclusão social}

A divulgação científica é vista como um elemento de inclusão social (MOREIRA, 2006), sendo uma das razões pelas quais muitos países têm estabelecido, nas últimas décadas, políticas e programas nacionais voltados para a popularização da Ciência e Tecnologia (C\&T), como ocorreu na Inglaterra, Estados Unidos, Chile, França e China.

No Brasil, em 2003, foi criado o Departamento de Popularização e Difusão da Ciência e Tecnologia - DEPDI na Secretaria de C\&T para Inclusão Social (SECIS) do então Ministério da Ciência e Tecnologia (MCT), hoje Ministério da Ciência, Tecnologia e Inovação (MCTI), tendo por finalidade subsidiar a formulação e a implementação de políticas, programas e a definição de estratégias para a popularização e para a difusão de conhecimentos científicos e tecnológicos nas diversas instâncias sociais, contando com a participação das instituições de ensino e pesquisa. Sendo a inclusão social uma das prioridades políticas da gestão do presidente da república Luís Inácio Lula da Silva, a popularização da C\&T adquire, desde seu primeiro mandato, um status de maior relevância.

Como destaca Ildeu de Castro Moreira (2006), que esteve à frente do DEPDI no período de 2004 a 2013, a inclusão social pode ser entendida não só como a ação de proporcionar oportunidades e condições de adequada qualidade de vida a populações carentes, como também, em um sentido mais amplo, envolve o estabelecimento de condições para o pleno exercício da cidadania, com acesso a conhecimentos, meios e mecanismos de participação política que capacitem os cidadãos a agir de forma fundamentada e consciente. Um dos aspectos da inclusão social é possibilitar que cada cidadão tenha a oportunidade de adquirir um conhecimento básico sobre a ciência e seu funcionamento, que proporcione condições de entender o seu entorno, de ampliar suas oportunidades no mercado de trabalho e de atuar politicamente com conhecimento de causa. Para a educação de qualquer cidadão no mundo contemporâneo, portanto, é fundamental que ele dis- 
ponha de informações tanto no que concerne à ciência e tecnologia, como sobre seus principais resultados, métodos, usos, riscos, limitações e também sobre os interesses e determinações (econômicas, políticas, militares, culturais etc.) que presidem seus processos e aplicações.

Além disso, a prática científica, com seu significado social e cultural, ocupa um espaço de interlocução entre a ciência e a sociedade. Embora a atividade científica, ao longo dos séculos, se profissionalize, se institucionalize, ganhando uma certa autonomia em relação a outras atividades sociais, econômicas e culturais, ela se dá, e sempre se deu, dentro da sociedade, e esta autonomia é apenas relativa. Ainda que as relações entre a esfera científica e outras esferas da sociedade tenham se alterado com o passar dos séculos, ainda que variem conforme a área de conhecimento, de tecnologia e do país em questão, o fato de ela jamais ser totalmente independente faz com que as interlocuções envolvidas em sua produção não se restrinjam exclusivamente ao campo dos especialistas (SILVA, 2006: 56). Falar de inclusão social no domínio da difusão ampla dos conhecimentos científicos e tecnológicos e de suas aplicações compreende, portanto, atingir não só as populações pobres, mas também outras parcelas da população que se encontram excluídas no que se refere a um conhecimento científico e tecnológico básico (MOREIRA, 2006).

Por isso é que o termo mais aceito para se referir ao público-alvo da divulgação científica é público "não especializado", e não "leigo". A interlocução, afinal, pode se dar também entre cientista e cientista.

\section{Ciência e democracia}

À luz da consideração de que a divulgação científica e tecnológica tem um papel importante na formação permanente de cada cidadão e no aumento da qualificação geral científico-tecnológica da sociedade, e, portanto, na inclusão social, não se pode deixar de reconhecer a relevância dessa área para o desenvolvimento democrático de um país.

Entre os objetivos centrais da popularização da C\&T estão suas contribuições para promover a melhoria e maior atualização/modernização do ensino das ciências em todos os níveis de ensino, com ênfase nas ações e atividades que deem a devida relevância e estimulem a criatividade, a experimentação e a interdisciplinaridade cultural e a valorização de conhecimentos populares e tradicionais, além de estimular e promover maior participação popular nas questões gerais de C\&T (MOREIRA, 2006). Nessa perspectiva, a divulga- 
EXPERIÊNCIAS EXEMPLARES DE DIVULGAÇÃO CIENTÍFICA DO CENTRO BRASILEIRO DE PESQUISAS FÍSICAS

ção científica e os processos de comunicação pública da ciência assumem hoje papel determinante no estímulo à participação cidadã e no resgate das vozes e percepções do público sobre temas de ciência e tecnologia.

\section{Expansão do campo científico}

Acredita-se que a divulgação científica promova um movimento de atração de jovens estudantes às carreiras científicas - e, por óbvio, uma expansão do número de cientistas implica a expansão do campo científico. Por outro lado, parece que os cientistas vêm percebendo que é mais provável conseguir financiamento para suas pesquisas na medida em que eles se tornam bons comunicadores - até porque entre os integrantes do público-alvo da divulgação científica, o público não especializado, podem figurar os gestores responsáveis pela captação e liberação de recursos para as pesquisas.

\section{A DIVULGAÇÃo CIENTÍFICA NO CBPF - BREVE HISTÓRICO}

Como a promoção da divulgação científica é uma de suas atribuições', o Centro Brasileiro de Pesquisas Físicas esteve, ao longo de sua história, atento à relevância das atividades nessa seara. Uma de suas publicações mais tradicionais, a revista Ciência e Sociedade, instituída por um dos fundadores do CBPF, o físico José Leite Lopes, veicula, desde sua primeira edição, em 1963, artigos sobre história da ciência, questões sociopolíticas, mas também textos que procuram aproximar o público não especializado de tópicos da pesquisa científica. Em 2013, ano em que comemorou 50 anos de existência, a publicação agregou o ISSN eletrônico ao seu escopo, (ISSN impresso: 0101-9228; ISSN eletrônico: 2317-4595), seus artigos passaram a ser divulgados na íntegra na página do CBPF, incorporando, também, o número de DOI (digital object identifier). ${ }^{2}$

Para além desse espaço, diversos pesquisadores da instituição atuaram, de forma mais ou menos consistente, na área da divulgação científica3. Em 2000, entretanto, com o lançamento da revista CBPF - Na Vanguarda

1 Art. $5^{\circ}$, inciso IV, da Portaria MCT n ${ }^{\circ} 638$, de 27 de setembro de 2007 (Regimento Interno do Centro Brasileiro de Pesquisas Físicas).

2 Essas informações e um panorama detalhado da revista Ciência e Sociedade podem ser encontrados em OTTONI, 2013.

3 Entre algumas iniciativas podemos destacar as atividades de Francisco Caruso Neto 
da Pesquisa, foi dado um passo significativo em direção a uma ação mais sistematizada e continuada voltada à divulgação científica. Com uma única edição até o momento, a publicação, idealizada pelo pesquisador João Carlos Costa dos Anjos, então diretor do CBPF, e tendo como editor o jornalista Cássio Leite Vieira (Ciência Hoje), apresentava em linguagem acessível ao público não especializado vários grupos de pesquisa da instituição e suas respectivas áreas. Ainda integravam a publicação as sessões "Tome Nota", com informações sobre infraestrutura, colaborações, formação de recursos humanos e produção científica do CBPF; "Um pouco de história", que apresentava brevemente fatos ligados à sua criação; "Lembranças de um fundador", com base em entrevista realizada com Cesar Lattes; "Sonho com a Física no Rio", com foco na atividade de José Leite Lopes; e "Ensino e Pesquisa como meta", elaborada a partir de depoimentos de Jayme Tiomno e Elisa Frota-Pêssoa, todos participantes da fundação do CBPF.

\section{PROJetos de divUlgaÇÃo CIENTÍFICA DO CBPF}

\subsection{Desafios da Física}

Em 2002, também sob a coordenação de João dos Anjos, teve início o projeto Desafios da Física. Segundo entrevista concedida por Anjos, o projeto teve como inspiração os trabalhos da Comissão instituída pelo Ministério da Ciência e Tecnologia para avaliar as atividades em Física no Brasil, da qual também foi um dos membros ${ }^{4}$. O Relatório apresentado pela Comissão destacava as áreas em que seria relevante a atuação das instituições de ensino

através do projeto "Tirinhas de Fisica” (http://www.cbpf.br/ caruso/tirinhas/index.htm) e com diversas publicações voltadas à divulgação científica; Henrique Lins de Barros com sua pesquisa sobre Santos Dumont; Ivan dos Santos Oliveira com o livro Física para Aficionados;e diversas publicações de Alberto Passos Guimarães, pesquisador emérito do CBPF, indicado para a direção do Instituto Ciência Hoje (ICH) em 2013, entidade da qual é um dos fundadores e que desenvolve atividades educativas e de divulgação científica, vinculada à Sociedade Brasileira para o Progresso da Ciência (SBPC). O ICH desempenha um importante papel na popularização e divulgação das atividades científicas no país, concentrando sua ação em três frentes principais: a publicação mensal das revistas Ciência Hoje e Ciência Hoje das Crianças, o portal CH-Online e os programas de apoio à educação, desenvolvidos em conjunto com escolas públicas do país.

4 A Comissão foi instituída através da Portaria ministerial no 51 de 22.01.2002. A íntegra do relatório está disponível em:<http://portal.cbpf.br/protected/Pages/o_cbpf/pdfs/RelatorioCFB-MCT.pdf>. 
e pesquisa não só no país, mas também no cenário mundials.

Com ênfase nas áreas destacadas, o primeiro folheto, intitulado 12 desafios da física para o século 21, com tiragem de três mil exemplares, foi feito para ser distribuído na Reunião Anual da Sociedade Brasileira para o Progresso da Ciência (SBPC) realizada no ano de 2002 em Goiânia. A publicação, financiada com recursos orçamentários do CBPF, apresentava alguns dos principais desafios que a física deveria enfrentar no século que acabava de se iniciar. Os doze temas eram: Raios Cósmicos de Altas Energias, Unificação das Forças da Natureza, Origem das Massas, Plasmas de Quark-Glúon, Ondas Gravitacionais, Computador Quântico, Manipulação de Átomos e os Novos Materiais, Matéria Escura, Energia Escura, Massa dos Neutrinos, Formação dos Elementos Pesados, A biofísica das proteínas e do DNA.

Com a excelente receptividade que o folheto obteve, a instituição resolveu dar continuidade ao projeto e, além de recursos de seu próprio orçamento, buscou financiamento do Conselho Nacional de Desenvolvimento Científico e Tecnológico - CNPq, da Fundação Carlos Chagas Filho de Amparo à Pesquisa do Estado do Rio de Janeiro - FAPERJ, e da extinta Fundação Vitae, uma associação civil voltada ao apoio à cultura, à educação e à promoção social.

A partir dos doze temas do folheto inaugural, surgiram: "Nanociência e Nanotecnologia - modelando o futuro átomo por átomo" (2003/ atualizado em 2006); "Sistemas complexos - a fronteira entre a ordem e o caos" (2003/atualizado em 2005); "Raios cósmicos - energias extremas no universo” (2004). Já sob a direção de Ricardo Galvão - diretor do CBPF de agosto de 2004 a junho de 2012 - e no bojo das celebrações do Ano Mundial da Física' foram publicados: “Ano Miraculoso de Einstein - 100 anos da publicação

5 Publicação recente do National Research Council, dos Estados Unidos da América (Physics in a New Era: An Overview, National AcademyPress, Washington, DC, 2001) lista seis áreas da Física, consideradas altamente prioritárias pelo grau de desafio e potencial de desenvolvimento que encerram. Essas áreas são: (i) Desenvolvimento de tecnologias quânticas; (ii) Sistemas complexos; (iii) Aplicação da Física na Biologia; (iv) Novos materiais; (v) Exploração do Universo; (vi) Unificação das forças da Natureza. É fundamental que nosso País reforce e desenvolva sua capacitação em todas essas áreas. Enfatizamos no entanto, na lista a seguir, quatro temas que, em nossa opinião, poderiam se beneficiar fortemente de um esforço coordenado por parte da comunidade científica e das agências de financiamento [Novos materiais, Nanociências, Tecnologias quânticas, Aplicação da Física na Biologia]. (2002: 32)

$6 \quad \mathrm{O}$ ano de 2005 foi escolhido pela International Union of Pure and Applied Physics (União Internacional de Física Pura e Aplicada) como o “Ano Mundial da Física”, propos- 
de artigos que mudaram a física" (2005); "Cosmologia - a busca pela origem, evolução e estrutura do universo" (2005); "Partículas elementares - a (des)construção da matéria pelo homem" (2005). "Biofísica - duas visões da vida" (2006); "Informação Quântica - do teleporte à ultima fronteira da computação" (2006); "Neutrinos - as misteriosas partículas-fantasma" (2006); "Supercordas - o sonho da unificação das quatro forças da natureza" (2006) e "LHC - o gigante criador de matéria" (2008) completaram a série inicial. Posteriormente foram publicados mais quatro folhetos: "Núcleo Atômico - 100 anos da descoberta do centro da matéria" (2011); "O Sistema Solar - nossa vizinhança cósmica" (2012); "Meteoritos - os mensageiros do espaço" (2011) e "Buracos Negros: devoradores cósmicos de matéria e luz" (2012). Com edição de texto de Cássio Leite Vieira, os folhetos da série contam com um ou mais editores científicos do CBPF - em sua maioria - e de universidades e instituições de ensino e pesquisa do Brasil.

Inicialmente distribuídos na própria instituição, na Expociência - promovida anualmente pela SBPC - e em outros encontros, feiras, exposições, com a criação do sítio "Desafios da Física - Projeto de Divulgação Científica" (www.cbpf.br desafiosindex_mphp=), os folhetos também foram disponibilizados no formato de arquivo eletrônico "pdf" e passaram a ser enviados pelo correio, atendendo solicitação via página eletrônica.

Em 2008, com o financiamento da Vitae e da Fundação Carlos Chagas FiIho de Amparo à Pesquisa do Estado do Rio de Janeiro (FAPERJ), foi editado o livro Um olhar para o Futuro - desafios da Física para o século 21. Integram a publicação onze capítulos, que correspondem aos folhetos publicados revisados e acrescidos de novas imagens.

Atualmente, estuda-se a possibilidade de revisão de conteúdo e reedição dos folhetos da série, muitos dos quais já se encontram esgotados.

\subsection{Algumas razões para ser um cientista - o livro}

Com a instituição, em 2004, da Semana Nacional de Ciência e Tecnologia (SNCT) pelo então Ministro de Estado da área, Eduardo Campos, o CBPF, as

ta que foi apoiada pela UNESCO e pela Assembleia Geral das Nações Unidas. Trata-se de um marco do centenário do annus mirabilis de Albert Einstein, isto é, de quando Einstein publicou seus trabalhos sobre o efeito fotoelétrico, a relatividade especial e o movimento browniano. Por todo o mundo foram organizados eventos e diversas atividades para comemorar o ano. No Brasil, a organização oficial coube à Sociedade Brasileira de Física (SBF). 
outras unidades de pesquisa do MCT e as instituições de ensino e pesquisa de todo o país passaram a desenvolver de maneira mais sistematizada e unificada diversas atividades que têm como objetivo aproximar ciência e sociedade, procurando, simultaneamente, desmitificar a pesquisa científica, prestar contas à sociedade quanto aos recursos públicos nela empregados e despertar e incentivar o interesse de crianças e jovens para a pesquisa científica e tecnológica. Na primeira edição da SNCT, o CBPF, além de apresentar projetos desenvolvidos na instituição nos eventos coordenados pela equipe do MCT no Rio de Janeiro, ofereceu atividades internas que compreenderam exposições, realização de experimentos, palestras voltadas ao público não especializado e visitas guiadas a seus laboratórios de pesquisa.

Foi em 2005, no ensejo do planejamento das atividades para a $2^{a}$ Semana Nacional de Ciência e Tecnologia, que teve início o projeto da publicação Algumas razões para ser um Cientista. Proposto pela direção do CBPF, e tendo como modelo a publicação One Hundred Reasons To Be a Scientist do Centro Internacional para a Física Teórica - ICTP’, a elaboração da obra foi financiada com recursos orçamentários do CBPF. Esse projeto reuniu traduções de alguns textos de físicos e matemáticos do livro do ICTP e depoimentos de expoentes brasileiros na pesquisa em Física, destacando-se por ter mobilizado os diversos setores institucionais na sua consecução: membros do corpo docente e discente foram responsáveis pela tradução dos textos selecionados; a física Carolina Cronemberger, então aluna de doutorado da instituição, realizou entrevistas com os cientistas brasileiros e as editou; e a supervisão do texto final e distribuição coube a servidores de gestão. O livro, que alcançou grande repercussão, pretende levar para mais perto da população um pouco da história, amálgama de experiência individual e vivência coletiva, daqueles que escolheram a ciência como profissão (mais do que isso, muitas vezes, como um modo de vida) e estimular novas vocações. Além de ser distribuído durante a Semana nos eventos externos e internos, o CBPF

7 One Hundred Reasons To Be a Scientist foi publicado no 400 aniversário do Abdus Salam International Centre for Theoretical Physics (ICTP). Em seu prefácio, lê-se: "Taking as the occasion the 4oth anniversary of our Centre, we thought it to be valuable to produce this book containing a number of brief and personal accounts by some of the most eminent scientists of our time, of what it was about science that captured their imagination as youngsters and kept it alive, and what main piece of knowledge they have added to the extraordinary lore of science. What message do they have for the budding scientists?" (2004:1). A utilização dos artigos selecionados para integrarem a publicação do CBPF foi autorizada pelo ICTP. 
atendeu, no primeiro ano da publicação, aproximadamente 500 pedidos $^{8}$, feitos via correio eletrônico, de remessa do livro, inclusive para o exterior.

Passados nove anos de seu lançamento, já em sua quinta reimpressão, com tiragens entre três e cinco mil exemplares cada, e contando com recursos da SECIS/MCTI, a instituição continua a atender pedidos de centenas de volumes de outros órgãos e instituições além de pedidos individuais do livro, demanda esta que varia mensalmente entre 15 a 20 exemplares. Essa procura se eleva em 100\%, aproximadamente, após a realização de eventos destinados à divulgação e popularização da ciência, em especial a Semana Nacional de Ciência e Tecnologia - tradicionalmente realizada em outubro - e a Expociência - realizada a cada ano em um estado da federação, no mês de julho -, durante os quais são distribuídos, em média, mil exemplares. Entre os solicitantes encontram-se estudantes do Ensino Médio e do Ensino Superior, professores e cidadãos de diversas regiões do país, bibliotecas, coordenações e secretarias municipais e estaduais de educação, e até de saúde, que se interessam pela publicação.

Em 2006, o CBPF recebeu o "Prêmio José Reis de Divulgação Científica", concedido pelo Conselho Nacional de Desenvolvimento Científico e Tecnológico - CNPq, pelos projetos Desafios da Física e Algumas razões para ser um Cientista, como reconhecimento à qualidade de seu trabalho de divulgação.

\subsection{LABDID - Laboratório Didático do CBPF}

Em 2005, por determinação do então Ministério da Ciência e Tecnologia, as unidades de pesquisa passaram por um intenso processo de planejamento estratégico, que contou com a participação de membros externos da comunidade científica, com vistas à elaboração do Plano Diretor da Unidade (PDU) para o período 2006-2011.

Alinhado com os resultados apontados nas atividades de prospecção e com o Plano de Ação do Ministério, o PDU 2006-2011 do CBPF incluiu como metas "Desenvolver instrumentos de demonstração que possam ser expos-

\footnotetext{
8 Esse número foi apurado com base nos registros de correio. As remessas do material de divulgação científica do CBPF são realizadas por sua Coordenação de Colaborações Científicas Institucionais - CCI/CBPF.

9 A partir de 2012 o Plano de Ação do Ministério foi substituído pelo documento intitulado Estratégia Nacional de Ciência, Tecnologia e Inovação 2012-2015- ENCTI, publicado em 2012.
} 
tos ao público leigo (terminologia hoje substituída por "público não especializado") em eventos destinados à popularização da ciência, como a "Semana Nacional de Ciência e Tecnologia", e "Estabelecer convênios que permitam a utilização de laboratórios e instalações do CBPF para a complementação da formação de estudantes do Ensino Médio e de graduação de instituições de ensino públicas.[...] Construir e equipar um laboratório de demonstrações até 2007." (CBPF, 2006: 32-33).

Em outubro de 2008, como parte das atividades do CBPF na SNCT, mas ainda em fase experimental, teve início o projeto Laboratório Didático do CBPF (LABDID) ${ }^{\circ 0}$ Com a primeira visita de estudantes para apresentação de experimentos de física por pesquisadores do instituto. O LABDID objetiva "promover o envolvimento do público não especializado e estudantes na discussão de conceitos da Física Moderna de forma empírica, relacionando-os com fatos do cotidiano, como a tecnologia, estimulando o pensamento abstrato e conclusões com base na metodologia científica e contribuindo assim para o esforço de inclusão social" ". Dispondo de equipamentos para a realização de vários experimentos, sua atuação se dá através de quatro frentes: 1) programa "Físico Por Uma Tarde", com a realização de visitas de escolas públicas de Ensino Médio ao CBPF; (2) desenvolvimento de experimentos com elevado teor de interatividade e importância histórica; (3) organização de programas de capacitação continuada de professores do Ensino Fundamental e Médio; e (4) participação do CBPF em eventos externos, levando experimentos de grande interatividade com o público. Essa última linha de ação é desenvolvida pelo Laboratório de Divulgação Científica (LABDIV)².

A maior parte dos experimentos são explorados nas visitas de escolas

10 A Portaria CBPF n 40, de 09 de agosto de 2007, designou uma Comissão de Atividades Didáticas de Extensão com o objetivo de "administrar o Laboratório Didático aberto à comunidade externa", integrada pelos pesquisadores Luiz Carlos Sampaio Lima (nomeado como presidente da comissão), Gilvan Augusto Alves, Sebastião Alves Dias e Martín Makler e pelo tecnologista Geraldo Roberto Carvalho Cernicchiaro. A Portaria $\mathrm{n}^{\circ} 04$, de 04 de fevereiro de 2010 passa a compreender nessa comissão o pesquisador André Massaferri Rodrigues, posteriormente alçado a presidente desse grupo pela Portaria CBPF $n^{\circ}$ 12, de 07 de maio de 2010.

11 Conforme descrição do programa disponível em: <http://portal.cbpf.br/index.php?page=FormacaoCientifica.labdid $>$ (acesso em 31.03.2014).

12 Embora com participação relevante em atividades externas de divulgação científica do CBPF, o LABDIV não conta ainda com uma regulamentação normativa institucional que especifique suas atribuições e funcionamento. 
no âmbito do programa "Físico Por Uma Tarde", pilar da atuação do LABDID. Ao final de cada ano são abertas inscrições para as 34 vagas disponíveis, 16 das quais reservadas a escolas estaduais do Rio de Janeiro. As visitas têm duração de 3 horas e meia, com horários pela manhã e tarde e compreendem três etapas: 1) uma conversa informal sobre Física, que apresenta também as principais linhas de pesquisa do Centro; 2) realização de experimentos e demonstrações pelos estudantes, orientados por monitores - os experimentos são divididos em quatro módulos temáticos: Luz, Elétron, Átomo e Aplicações - e visita em grupos a três laboratórios do CBPF, previamente selecionados, com o intuito de visualizar o cotidiano de uma instituição de pesquisa. Até 2011 o Programa recebia aproximadamente 550 estudantes de nível médio por ano. A partir de 2012, com sua instalação definitiva, passou a receber 1000 estudantes por ano. A demanda de participações no programa vem aumentando substancialmente, a ponto de não ser possível a visitação de turmas no mesmo ano de sua inscrição, devido ao rápido esgotamento da agenda anual aberta para a atividade.

\section{CONSIDERAÇÕES FINAIS}

As diversas edições e o incremento das solicitações dos folhetos e de exemplares do livro após sua distribuição em eventos de grande porte, como a SNCT e a Expociência, e a impossibilidade de atender em sua totalidade as inscrições nos programas desenvolvidas pelo LABDID comprovam o interesse suscitado pela Ciência e indicam que a atuação das instituições de ensino e pesquisa do país na área de divulgação científica, além de sua relevância ímpar, está longe de se esgotar.

Nesse sentido, as três principais experiências de divulgação científica do CBPF apresentadas - Projeto Desafios da Física, livro Algumas razões para ser um cientista e Laboratório Didático (LABDID) - são exemplares na medida em que, com base na verificada grande demanda social dos trabalhos desenvolvidos pelo corpo de excelência dos cientistas envolvidos, se mostram bem sucedidas no atingimento do escopo de levar conhecimento científico ao público não especializado.

Por outro lado, estão em consonância com os esforços de política científica dos países democráticos em geral, que têm dado cada vez mais importância à divulgação científica, inclusive o Brasil, cuja Estratégia Nacional de Ciência, Tecnologia e Inovação 2012-2015 compreende o objetivo de desenvolver e difundir conhecimento e soluções criativas para a inclusão produ- 
EXPERIÊNCIAS EXEMPLARES DE DIVULGAÇÃO CIENTÍFICA DO CENTRO BRASILEIRO DE PESQUISAS FÍSICAS

tiva e social, a melhoria da qualidade de vida e o exercício da cidadania no programa prioritário de C,T\&I para o desenvolvimento social.

\section{REFERÊNCIAS}

ANJOS, João Carlos Costa dos; VIEIRA, Cássio Leite. Um olhar para o futuro. Rio de Janeiro: Vieira \& Lent, 2009.

ANDRADE, Ana Maria Ribeiro de. Físicos, mésons e política: a dinâmica da ciência na sociedade. Rio de Janeiro: Hucitec, Museu de Astronomia e Ciências Afins, 1999.

CHAVES, A. et alli. Relatório Apresentado ao Ministério da Ciência e Tecnologia Sobre Alguns Aspectos da Física Brasileira, 2002. Disponível em:

<http://portal.cbpf.br/protected/Pages/o_cbpf/pdfs/RelatorioCFB-MCT.pdf>.

CBPF - Na vanguarda da pesquisa. Rio de Janeiro: CBPF, 2000.

Estratégia Nacional de Ciência, Tecnologia e Inovação - ENCTI 2012-2015.

LEITÃO, Pedro; ALBAGLI, Sarita. Popularización de la ciencia y la tecnología: una revisión de literatura. In: Martinez, E. ; Florez, J. (comp.). La popularización de la ciencia y la tecnología. México: FCE-Unesco-Red-POP FCE, 1997. p.17-37.

MASSARANI, Luiza et al. Ciência e Público: caminhos da divulgação científica no Brasil. Rio de Janeiro: Casa da Ciência - Centro Cultural de Ciência e Tecnologia da Universidade Federal do Rio de Janeiro, 2002.

MOREIRA, Ildeu de Castro. $\boldsymbol{A}$ inclusão social e a popularização da ciência e tecnologia no Brasil. Revista Inclusão Social, Vol. 1, n. 2 (2006), IBICT.

SILVA, Henrique César da. O que é divulgação científica? Revista Ciência \& Ensino, vol. 1, n. 1, 2006.

SILVA, Márcia Rocha da.; CARNEIRO, Maria Helena da Silva. Popularização da Ciência: análise de uma situação não formal de ensino. In: Reunião Anual da Associação Nacional de Pós-Graduação e Pesquisa em Educação (ANPEd), 29., 2006, Caxambu, MG. Trabalhos... Rio de Janeiro: ANPEd, 2006.

One Hundred Reasons to Be a Scientist. The ICTP Publications \& Printing Section. Trieste, 2004.

OTTONI, Heloísa Maria. "A Revista Ciência e Sociedade em Foco nestes últimos 50 anos (1963-2013)". In: Ciência e Sociedade. Rio de Janeiro: CBPF, v.1, n.1, 2013.

Plano diretor do CBPF, 2006 - 2010: planejamento estratégico do CBPF. Rio de Janeiro: CBPF, 2006.

Plano diretor do CBPF, 2011 - 2015: planejamento estratégico do CBPF. Rio de Janeiro: CBPF, 2011. 
Márcia de Oliveira Reis Brandão

Analista em C\&T Sênior do CBPF/MCTI. Doutora em Literatura Comparada (UFF), RJ.

\section{Larissa Santiago Ormay}

Analista em C\&T Pleno do CBPF/MCTI.

Doutoranda em Ciência da Informação (IBICT-UFRJ), RJ. 